\title{
Dark Spinors Hawking Radiation in String Theory Black Holes
}

\author{
R. T. Cavalcanti ${ }^{1,2}$ and Roldão da Rocha ${ }^{3,4}$ \\ ${ }^{1}$ CCNH, Universidade Federal do ABC, 09210-580 Santo André, SP, Brazil \\ ${ }^{2}$ Dipartimento di Fisica e Astronomia, Università di Bologna, Via Irnerio 46, 40126 Bologna, Italy \\ ${ }^{3}$ CMCC, Universidade Federal do ABC, 09210-580 Santo André, SP, Brazil \\ ${ }^{4}$ International School for Advanced Studies (SISSA), Via Bonomea 265, 34136 Trieste, Italy \\ Correspondence should be addressed to Roldão da Rocha; roldao.rocha@ufabc.edu.br
}

Received 26 August 2015; Accepted 24 November 2015

Academic Editor: Enrico Lunghi

Copyright (C) 2016 R. T. Cavalcanti and R. da Rocha. This is an open access article distributed under the Creative Commons Attribution License, which permits unrestricted use, distribution, and reproduction in any medium, provided the original work is properly cited. The publication of this article was funded by SCOAP ${ }^{3}$.

\begin{abstract}
The Hawking radiation spectrum of Kerr-Sen axion-dilaton black holes is derived, in the context of dark spinors tunnelling across the horizon. Since a black hole has a well defined temperature, it should radiate in principle all the standard model particles, similar to a black body at that temperature. We investigate the tunnelling of mass dimension one spin-1/2 dark fermions, which are beyond the standard model and are prime candidates to the dark matter. Their interactions with the standard model matter and gauge fields are suppressed by at least one power of unification scale, being restricted just to the Higgs field and to the graviton likewise. The tunnelling method for the emission and absorption of mass dimension one particles across the event horizon of Kerr-Sen axiondilaton black holes is shown here to provide further evidence for the universality of black hole radiation, further encompassing particles beyond the standard model.
\end{abstract}

\section{Introduction}

Black hole tunnelling procedures have been placed as prominent methods to calculate the temperature of black holes [19]. Tunnelling methods provide models for describing the black hole radiation. Various types of black holes have been investigated in the context of tunnelling of fermions and bosons as well $[1,2,9,10]$. Complementarily to the first results that studied the tunnelling of particles across black holes $[1,2]$, the Hamilton-Jacobi method was employed [3] and further generalized, by applying the WKB approximation to the Dirac equation [10]. Tunnelling procedures are quite used to investigate black holes radiation, by taking into account classically forbidden paths that particles go through, from the inside to the outside of black holes. Moreover, quantum WKB approaches were employed to calculate corrections to the Bekenstein-Hawking entropy for the Schwarzschild black hole [11].

The tunnelling method was employed to provide Hawking radiation due to dark spinors for black strings [12]. Moreover, this method was also employed to model the emission of spin-1/2 fermions, and the Hawking radiation was deeply analyzed as the tunnelling of Dirac particles throughout an event horizon, where quantum corrections in the single particle action are proportional to the usual semiclassical contribution. In addition, the modifications to the Hawking temperature and Bekenstein-Hawking entropy were derived for the Schwarzschild black hole. When spin-1/2 fermions are taken into account, the effect of the spin of each type of fermion cancels out, due to particles with the spin in any direction. Hence, the lowest WKB order implies that the black hole intrinsic angular momentum remains constant in tunnelling processes. Hawking radiation emulates semiclassical quantum tunnelling methods, wherein the HamiltonJacobi method is comprehensively used [3]. Mass dimension $3 / 2$ fermions tunnelling has been studied in the charged dilatonic black hole, the rotating Einstein-Maxwell-DilatonAxion black hole, and the rotating Kaluza-Klein black hole likewise [13]. For a review see [9].

Our approach here is to employ an exact classical solution in the low energy effective field theory describing heterotic string theory: the Kerr-Sen axion-dilaton black holes [14]. 
They present charge, magnetic dipole moment, and angular momentum, involving the antisymmetric tensor field coupled to the Chern-Simons 3-form. A myriad of black holes has been considered for tunnelling methods of fermions and bosons, as rotating and accelerating black holes and topological, BTZ, Reissner-Nordström, Kerr-Newman, and TaubNUT-AdS black holes, including the tunnelling of higher spin fermions as well [15].

We shall study similar methods for spin-1/2 fermions of mass dimension one, different from the procedure for standard mass dimension 3/2 fermions. Elko dark spinors, namely, the dual-helicity eigenspinors of the charge conjugation operator $[16,17]$, are spin-1/2 fermions of mass dimension one, with novel features that make them capable of incorporating both the Very Special Relativity (VSR) paradigm and the dark matter description as well $[16,18]$. Such spinors seem to be indeed a tip of the iceberg for a comprehensive class of nonstandard (singular) spinors [19, 20]. Moreover, a mass generation mechanism has been introduced in [21] for such dark particles, by a natural coupling to the kink solution in field theory. It provides exotic couplings among scalar field topological solutions and Elko dark spinors [21, $22]$. Due to its very small coupling with the standard model fields, except the Higgs field, dark spinors supply natural self-interacting dark matter prime candidates. Except for scalar fields and gravity, Elko dark spinors interactions with the standard model matter and gauge fields are suppressed by at least one power of unification/Planck scale [16]. In fact, the Lagrangian of such a field contains a quartic selfinteraction term and the interaction term of the new field with spin-zero bosonic fields. Moreover, Elko framework is shown to be invariant under the action of the HOM(2) VSR group and covariant under SIM(2) VSR group [23]. Elko dark spinor field is a representative of mass dimension one spin$1 / 2$ fermions in the type- 5 spinor field class in Lounesto's spinors classification; however, it is not the most general, since Majorana spinors are also encompassed by such class [22]. Some attempts to detect Elko at the LHC have been proposed, and important applications to cosmology have been widely investigated as well [12, 16, 18, 24-26].

This paper is presented as follows: the Kerr-Sen axiondilaton black hole is briefly revisited in the next section, together with the dark spinors framework. We thus shall calculate in Section 3 the probabilities of emission and absorption of Elko dark particles across these black holes. Therefore, the WKB approximation is used to compute the tunnelling rate and thus the resulting tunnelling probability. Finally the associated Hawking temperature shall be obtained, corroborating the universal character of the Hawking effect and further extending it to particles beyond the standard model.

\section{Kerr-Sen Axion-Dilaton Black Holes and Dark Spinors}

String theory has solutions describing extra-dimensional extended objects surrounded by event horizons, presenting a causal structure associated with singularities in string theory.
The low energy effective action of the heterotic string theory is ruled by an action that, up to higher derivative terms and other fields which are set to zero for the particular class of backgrounds considered [14], is given by

$$
\begin{aligned}
S= & -\int d^{4} x \sqrt{-\operatorname{det} G} e^{-\Phi} \\
& \cdot\left(-R+\frac{1}{12} H_{\mu \nu \rho} H^{\mu \nu \rho}-G^{\mu \nu} \partial_{\mu} \Phi \partial_{\nu} \Phi+\frac{1}{8} F_{\mu \nu} F^{\mu \nu}\right),
\end{aligned}
$$

where $G_{\mu \nu}$ is the metric regarding a $\sigma$-model [14], related to the Einstein metric by $e^{-\Phi} G_{\mu \nu}$, $\Phi$ denotes the dilaton field, $R$ stands for the scalar curvature, $F_{\mu \nu}=\partial_{[\mu} A_{\nu]}$ is the Maxwell field strength, and $H_{\mu \nu \rho}=\partial_{\mu} B_{\nu \rho}+\partial_{\rho} B_{\mu \nu}+\partial_{\nu} B_{\rho \mu}-\Omega_{\mu \nu \rho}$, for the Chern-Simons 3-form $\Omega_{\mu \nu \rho}-(1 / 4) A_{(\mu} F_{\nu \rho)}$. The above action can be led to the one in [27], up to the $H_{\mu \nu \rho} H^{\mu \nu \rho}$ term, after field redefinition.

The Kerr-Sen dilaton-axion black hole metric is a solution of the field equations derived from (1). In Boyer-Lindquist coordinates it reads

$$
\begin{aligned}
d s^{2}= & -\frac{\Delta-a^{2} \sin ^{2} \theta}{\Sigma} d t^{2}+\frac{\Sigma}{\Delta} d r^{2}+\Sigma d \theta^{2} \\
& +\frac{\sin ^{2} \theta}{\Sigma}\left[\left(r^{2}-2 \beta r-a^{2}\right)^{2}-\Delta a^{2} \sin ^{2} \theta\right] d \varphi^{2} \\
& -\frac{2 a \sin ^{2} \theta}{\Sigma}\left[\left(r^{2}-2 \beta r-a^{2}\right)^{2}-\Delta\right] d t d \varphi,
\end{aligned}
$$

where

$$
\begin{aligned}
& \Sigma=r^{2}-2 \beta r+a^{2} \cos ^{2} \theta \\
& \Delta=r^{2}-2 \eta r+a^{2}=\left(r-r_{+}\right)\left(r-r_{-}\right), \\
& \beta=\eta \sinh ^{2} \frac{\alpha}{2}
\end{aligned}
$$

and $r_{+}\left[r_{-}\right]$are the coordinate outer [inner] singularities.

Metric (2) describes a black hole solution with charge $Q$, mass $M$, magnetic dipole moment $\mu$, and angular momentum $J$, given by

$$
\begin{aligned}
& Q=\frac{\eta}{\sqrt{2}} \sinh \alpha, \\
& M=\frac{\eta}{2}(1+\cosh \alpha), \\
& \mu=\frac{1}{\sqrt{2}} \eta a \sinh \alpha, \\
& J=\frac{\eta a}{2}(1+\cosh \alpha) .
\end{aligned}
$$


The associated $g$-factor can be expressed as $g=2 \mu M / Q J=2$ [27]. The parameters can be expressed in terms of genuinely physical quantities as

$$
\begin{aligned}
& \eta=M-\frac{Q^{2}}{2 M}, \\
& \alpha=\operatorname{arcsinh}\left(\frac{2 \sqrt{2} Q M}{2 M^{2}-Q^{2}}\right), \\
& a=\frac{J}{M} .
\end{aligned}
$$

The coordinate singularities thus read $r_{ \pm}=M-Q^{2} / 2 M \pm$ $\sqrt{-J^{2} / M^{2}+\left(M-Q^{2} / 2 M\right)^{2}}$ which vanishes unless $|J|<$ $M^{2}-Q^{2} / 2$. The area of the outer event horizon is given by

$$
A=8 \pi M\left(M-\frac{Q^{2}}{2 M}+\sqrt{-\frac{J^{2}}{M^{2}}+\left(M-\frac{Q^{2}}{2 M}\right)^{2}}\right) .
$$

Thus, in the extremal limit, since $|J| \rightarrow M-Q^{2} / 2 M$, it reads $A \rightarrow 8 \pi|J|$. In this limit the horizon is hence finite and the surface gravity $\kappa$ or, equivalently, the Hawking temperature $T_{H}=\kappa / 2 \pi$ is provided by [14]

$$
\kappa=\frac{\sqrt{-4 J^{2}+\left(2 M^{2}-Q^{2}\right)^{2}}}{2 M\left(2 M^{2}-Q^{2}+\sqrt{-4 J^{2}+\left(2 M^{2}-Q^{2}\right)^{2}}\right)} .
$$

Thus in the extremal limit we have the limit $\kappa \rightarrow 0$ if $J \neq 0$. On the other hand, if $J=0$ then $\kappa=1 / 4 M$, in agreement with the results of $[27,28]$. For $J \neq 0$ this black hole solution has aspects analogous to the extremal rotating black hole rather than extremal charged black holes [27].

By performing the transformation $\phi=\varphi-\Omega t$, where $\Omega=$ $a\left(a^{2}-2 \beta r+a^{2}-\Delta\right) /\left(\left(r^{2}-2 \beta r+a^{2}\right)^{2}-\Delta a^{2} \sin ^{2} \theta\right)$, the metric (2) takes the form

$$
\begin{aligned}
d s^{2}= & -\frac{\Delta \Sigma}{\left(r^{2}-2 \beta r-a^{2}\right)^{2}-\Delta a^{2} \sin ^{2} \theta} d t^{2}+\frac{\Sigma}{\Delta} d r^{2} \\
& +\Sigma d \theta^{2} \\
& +\frac{\sin ^{2} \theta}{\Sigma}\left[\left(r^{2}-2 \beta r-a^{2}\right)^{2}-\Delta a^{2} \sin ^{2} \theta\right] d \phi^{2} .
\end{aligned}
$$

To study the Hawking radiation at the event horizon, the metric is regarded near the horizon:

$$
\begin{aligned}
d s^{2}= & -F\left(r_{+}\right) d t^{2}+\frac{1}{G\left(r_{+}\right)} d r^{2}+\Sigma\left(r_{+}\right) d \theta^{2} \\
& +\frac{H\left(r_{+}\right)}{\sum\left(r_{+}\right)} d \phi^{2},
\end{aligned}
$$

where

$$
\begin{aligned}
& H\left(r_{+}\right)=\sin ^{2} \theta\left(r_{+}^{2}-2 \beta r_{+}+a^{2}\right)^{2}, \\
& F\left(r_{+}\right)=\frac{2\left(r_{+}-\eta\right)\left(r-r_{+}\right) \Sigma\left(r_{+}\right)}{\left(r_{+}^{2}-2 \beta r_{+}+a^{2}\right)^{2}}, \\
& G\left(r_{+}\right)=\frac{2\left(r_{+}-\eta\right)\left(r-r_{+}\right)}{\Sigma\left(r_{+}\right)} .
\end{aligned}
$$

In order to analyze the tunnelling of Elko dark particles across the Kerr-Sen black hole event horizon, we will study the role that Elko dark particles play in this background. The essential prominent Elko particles features are in short revisited [16]. Elko dark spinors $\lambda\left(p^{\mu}\right)$ are eigenspinors of the charge conjugation operator $C$; namely, $C \lambda\left(p^{\mu}\right)= \pm \lambda\left(p^{\mu}\right)$. The plus [minus] sign regards self-conjugate [anti-self-conjugate] spinors, denoted by $\lambda^{S}\left(p^{\mu}\right)\left[\lambda^{A}\left(p^{\mu}\right)\right]$. For spinors at rest $\lambda\left(k^{\mu}\right)$ the boosted spinors read $\lambda\left(p^{\mu}\right)=e^{i \kappa \cdot \varphi} \lambda\left(k^{\mu}\right)$, where $k^{\mu}=$ $\left(m, \lim _{p \rightarrow 0} \mathbf{p} /|\mathbf{p}|\right)$, where $e^{i \boldsymbol{\kappa} \cdot \boldsymbol{\varphi}}$ denotes the boost operator. $\phi\left(k^{\mu}\right)$ are defined to be eigenspinors of the helicity operator, as $\boldsymbol{\sigma} \cdot \widehat{\mathbf{p}} \phi^{ \pm}\left(k^{\mu}\right)= \pm \phi^{ \pm}\left(k^{\mu}\right)$, where [16]

$$
\begin{aligned}
& \phi^{+}\left(k^{\mu}\right)=\sqrt{m}\left(\begin{array}{c}
\cos \left(\frac{\theta}{2}\right) e^{-i \varphi / 2} \\
\sin \left(\frac{\theta}{2}\right) e^{+i \varphi / 2}
\end{array}\right) \equiv\left(\begin{array}{c}
\alpha \\
\beta
\end{array}\right), \\
& \phi^{-}\left(k^{\mu}\right)=\sqrt{m}\left(\begin{array}{c}
-\sin \left(\frac{\theta}{2}\right) e^{-i \varphi / 2} \\
\cos \left(\frac{\theta}{2}\right) e^{+i \varphi / 2}
\end{array}\right)=\left(\begin{array}{c}
-\beta^{*} \\
\alpha^{*}
\end{array}\right) .
\end{aligned}
$$

Elko dark spinors $\lambda\left(k^{\mu}\right)$ are constructed as

$$
\begin{aligned}
& \lambda_{ \pm}^{S}\left(k^{\mu}\right)=\left(\begin{array}{c}
\sigma_{2}\left(\phi^{ \pm}\left(k^{\mu}\right)\right)^{*} \\
\phi^{ \pm}\left(k^{\mu}\right)
\end{array}\right), \\
& \lambda_{ \pm}^{A}\left(k^{\mu}\right)= \pm\left(\begin{array}{c}
-\sigma_{2}\left(\phi^{\mp}\left(k^{\mu}\right)\right)^{*} \\
\phi^{\mp}\left(k^{\mu}\right)
\end{array}\right)
\end{aligned}
$$

and have dual helicity, as $-i \sigma_{2}\left(\phi^{ \pm}\right)^{*}$ has helicity dual to that of $\phi^{ \pm}$. The boosted terms

$$
\begin{aligned}
& \lambda_{ \pm}^{A}\left(p^{\mu}\right)=\sqrt{\frac{E+m}{2 m}}\left(1 \pm \frac{p^{\mu}}{E+m}\right) \lambda_{ \pm}^{A}, \\
& \lambda_{ \pm}^{S}\left(p^{\mu}\right)=\sqrt{\frac{E+m}{2 m}}\left(1 \mp \frac{p^{\mu}}{E+m}\right) \lambda_{ \pm}^{S}
\end{aligned}
$$

are the expansion coefficients of a mass dimension one quantum field. The Dirac operator does not annihilate the $\lambda\left(p^{\mu}\right)$, but instead the equations of motion read $[16,17]$

$$
\begin{aligned}
& \gamma_{\mu} \nabla^{\mu} \lambda_{ \pm}^{S}= \pm i \frac{m}{\hbar} \lambda_{\mp}^{S}, \\
& \gamma_{\mu} \nabla^{\mu} \lambda_{\mp}^{A}= \pm i \frac{m}{\hbar} \lambda_{ \pm}^{A}
\end{aligned}
$$

Dark spinors nevertheless satisfy the Klein-Gordon equation. 
A mass dimension one quantum field can be thus constructed as [17]

$$
\begin{aligned}
\mathfrak{f}(x) & =\int \frac{d^{3} p}{(2 \pi)^{3}} \frac{1}{\sqrt{2 m E(\mathbf{p})}} \\
& \cdot \sum_{\rho}\left[b_{\rho}^{\dagger}(\mathbf{p}) \lambda^{A}(\mathbf{p}) e^{i p_{\mu} x^{\mu}}+a_{\rho}(\mathbf{p}) \lambda^{S}(\mathbf{p}) e^{-i p_{\mu} x^{\mu}}\right] .
\end{aligned}
$$

The creation and annihilation operators $a_{\rho}(\mathbf{p}), a_{\rho}^{\dagger}(\mathbf{p})$ satisfy the Fermi statistics [17], with similar anticommutators for $b_{\rho}(\mathbf{p})$ and $b_{\rho}^{\dagger}(\mathbf{p})$. The mass dimensionality of $\mathfrak{f}(x)$ can be realized from the adjoint

$$
\begin{aligned}
& \mathfrak{f}(x)=\int \frac{d^{3} \mathbf{p}}{(2 \pi)^{3}} \frac{1}{\sqrt{2 m E(\mathbf{p})}} \\
& \left.\quad \cdot \sum_{\rho}\left[b_{\rho}(\mathbf{p}) \neg^{A}(\mathbf{p}) e^{-i p_{\mu} x^{\mu}}+a_{\rho}^{\dagger}(\mathbf{p})\right\urcorner^{S}(\mathbf{p}) e^{i p_{\mu} x^{\mu}}\right],
\end{aligned}
$$

where, denoting hereupon by $\sigma^{\mu}$ the Pauli matrices, the conjugate spinors are constructed by $\vec{\lambda}_{\rho}\left(p^{\mu}\right)=\left[\Xi \lambda_{\rho}\left(p^{\mu}\right)\right]^{\dagger} \sigma_{1} \otimes$ $\mathbb{q}_{2}$. Here the operator $\Xi=(1 / 2 m)\left(\lambda_{-}^{S}\left(p^{\mu}\right) \bar{\lambda}_{-}^{S}\left(p^{\mu}\right)_{-}\right.$ $\left.\lambda_{-}^{A}\left(p^{\mu}\right) \bar{\lambda}_{-}^{A}\left(p^{\mu}\right)+\lambda_{+}^{S}\left(p^{\mu}\right) \bar{\lambda}_{+}^{S}\left(p^{\mu}\right)-\lambda_{+}^{A}\left(p^{\mu}\right) \bar{\lambda}_{+}^{A}\left(p^{\mu}\right)\right)$ is an involution [17], where the standard Dirac conjugate $\bar{\lambda}\left(p^{\mu}\right)=$ $\lambda\left(p^{\mu}\right)^{\dagger} \gamma^{0}$ is adopted. The mass dimension of the new field is determined by the $\operatorname{SIM}(2)$ covariant propagator [17]

$$
\begin{aligned}
& S\left(x-x^{\prime}\right)=i\left\langle\left|\mathrm{~T}\left(\mathfrak{f}(x) \mathfrak{f}\left(x^{\prime}\right)\right)\right|\right\rangle \\
& \quad=-\lim _{\epsilon \rightarrow 0^{+}} \int \frac{d^{4} p}{(2 \pi)^{4}} e^{-i p^{\mu}\left(x_{\mu}^{\prime}-x_{\mu}\right)}\left(\frac{\square+\mathscr{G}(\varphi)}{p^{\mu} p_{\mu}-m^{2}+i \epsilon}\right),
\end{aligned}
$$

where $\mathrm{T}$ is the canonical time-ordering operator and

$$
\mathscr{G}(\varphi)=\left(\begin{array}{cc}
0 & -i e^{-i \varphi} \\
i e^{i \varphi} & 0
\end{array}\right) \otimes \sigma_{1}
$$

that respects symmetries of the theory of VSR [17, 23].

\section{Hawking Radiation from Tunnelling Dark Spinors}

Hawking radiation from general black holes encompasses distinguished charged and uncharged particles. Tunnelling methods can be employed for Elko dark particles across the horizon of Kerr-Sen black holes. From (8) the associated tetrad can be chosen so that the following generators can be achieved:

$$
\begin{aligned}
& \gamma^{t}=\frac{1}{\sqrt{F\left(r_{+}\right)}}\left(\begin{array}{cc}
0 & \mathbb{I}_{2} \\
\mathbb{I}_{2} & 0
\end{array}\right), \\
& \gamma^{\theta}=\frac{1}{\sqrt{\sum\left(r_{+}\right)}}\left(\begin{array}{cc}
0 & \sigma^{2} \\
-\sigma^{2} & 0
\end{array}\right), \\
& \gamma^{r}=\sqrt{G\left(r_{+}\right)}\left(\begin{array}{cc}
0 & \sigma^{1} \\
-\sigma^{1} & 0
\end{array}\right), \\
& \gamma^{z}=\sqrt{\frac{\sum\left(r_{+}\right)}{H\left(r_{+}\right)}}\left(\begin{array}{cc}
0 & \sigma^{3} \\
-\sigma^{3} & 0
\end{array}\right) .
\end{aligned}
$$

Elko dark spinors can be written as

$$
\lambda_{+}^{S}=\left(\begin{array}{c}
-i \beta^{*} \\
i \alpha^{*} \\
\alpha \\
\beta
\end{array}\right) \exp \left(\frac{i}{\hbar} \widetilde{I}\right)
$$

$$
\lambda_{-}^{S}=\left(\begin{array}{c}
-i \alpha \\
-i \beta \\
-\beta^{*} \\
\alpha^{*}
\end{array}\right) \exp \left(\frac{i}{\hbar} \widetilde{I}\right)
$$

$$
\lambda_{+}^{A}=\left(\begin{array}{c}
i \alpha \\
i \beta \\
-\beta^{*} \\
\alpha^{*}
\end{array}\right) \exp \left(\frac{i}{\hbar} \widetilde{I}\right)
$$

$$
\lambda_{-}^{A}=\left(\begin{array}{c}
-i \beta^{*} \\
i \alpha^{*} \\
-\alpha \\
-\beta
\end{array}\right) \exp \left(\frac{i}{\hbar} \widetilde{I}\right)
$$

where $\widetilde{I}=\widetilde{I}(t, r, \theta, z)$ represents the classical action. We use the above forms for dark particles in each of (14) and (15) and then solve this coupled system of equations. By denoting

$$
\nabla_{\mu}=\partial_{\mu}+\frac{1}{8} i \Gamma_{\mu}^{\alpha \beta}\left[\gamma^{\alpha}, \gamma^{\beta}\right]
$$

where $\gamma^{\sigma}$ are the usual Clifford bundle generators for the Minkowski spacetime. By identifying $\lambda[\dot{\lambda}]$ to the Elko spinor on the left [right] hand side of (14) and (15), then (14) reads $\gamma^{\mu}\left(\nabla_{\mu}+e A_{\mu}\right) \lambda=i(m / \hbar) \dot{\lambda}$. Using the WKB approximation, where $\widetilde{I}=I+\mathcal{O}(\hbar)$, it yields

$$
\left(I_{\mu}+e A_{\mu}\right) \gamma^{\mu} \lambda=i m \dot{\lambda}+\mathcal{O}(\hbar)
$$


where $I_{\mu} \equiv \partial I / \partial x^{\mu}$. Taking merely the leading order terms in the above equation, from a general form $\lambda=(a, b, c, d)^{\top}$, $\grave{\lambda}=(\stackrel{\circ}{a}, \stackrel{b}{c}, \stackrel{\circ}{c}, \stackrel{d}{d})^{\top}$, we have general Elko dynamic equations governed by (23). The ansatz $I(t, r, \theta, \varphi)=-(\omega-j \Omega) t+j \varphi+$ $W(r)+\Theta(\theta)$ can be used, where $\omega$ and $j$ denote the energy and magnetic quantum number of the particles, respectively. Moreover, the parameters $a, b, c, d$ are not independent. In fact, (21) assert that for the self-conjugate spinors $\lambda^{S}$ we have $a=-i d^{*}$ and $b=i c^{*}$, whereas for the anti-selfconjugate spinors $\lambda^{A}$ it reads $a=i d^{*}$ and $b=-i c^{*}$. Thus, by corresponding the $\lambda^{S}\left[\lambda^{A}\right]$ spinors to the upper [lower] sign below, after awkward computation (23) yields

$$
\begin{aligned}
& \pm \sqrt{G\left(r_{+}\right)} W^{\prime} d^{*} \mp \frac{\left(\omega-j \Omega_{H}+e A_{+}\right)}{\sqrt{F\left(r_{+}\right)}} c^{*}=\stackrel{\circ}{d} m, \\
& \pm \sqrt{G\left(r_{+}\right)} W^{\prime} c^{*} \mp \frac{\left(\omega-j \Omega_{H}+e A_{+}\right)}{\sqrt{F\left(r_{+}\right)}} d^{*}=-\stackrel{c}{ } m .
\end{aligned}
$$

The angular function $j \varphi+\Theta(\theta)$ must be a complex function and the same solution for it is achieved for both incoming and outgoing cases as well. It implies that the contribution of such function vanishes after dividing the outgoing probability by the incoming one. Hence the angular function can be neglected hereupon.

In the above system the equations for $\lambda_{+}^{S}\left[\lambda_{-}^{S}\right]$ are shown to be equivalent to the ones for $\lambda_{-}^{A}\left[\lambda_{+}^{A}\right]$. Thus we have to deal solely with the self-conjugate $\lambda_{+}^{S}$ spinors. Moreover, there are more underlying equivalences. In fact, (24) for $\lambda_{ \pm}^{S}$ is equivalent to (25) for $\lambda_{\mp}^{S}$. Consequently there is just a couple of equations for $\lambda_{ \pm}^{S}$ given by

$$
\begin{gathered}
\sqrt{G\left(r_{+}\right)} W^{\prime} \alpha^{*}-\frac{\left(\omega-j \Omega_{H}+e A_{+}\right)}{\sqrt{F\left(r_{+}\right)}} \beta^{*}=\beta^{*} m, \\
\sqrt{G\left(r_{+}\right)} W^{\prime} \beta+\frac{\left(\omega-j \Omega_{H}+e A_{+}\right)}{\sqrt{F\left(r_{+}\right)}} \alpha=\alpha m, \\
\sqrt{G\left(r_{+}\right)} W^{\prime} \alpha \mp \frac{\left(\omega-j \Omega_{H}+e A_{+}\right)}{\sqrt{F\left(r_{+}\right)}} \beta=\mp \beta m, \\
\sqrt{G\left(r_{+}\right)} W^{\prime} \beta^{*} \mp \frac{\left(\omega-j \Omega_{H}+e A_{+}\right)}{\sqrt{F\left(r_{+}\right)}} \alpha^{*}= \pm \alpha^{*} m .
\end{gathered}
$$

Combining either (26) or (27) implies equations for either $\lambda_{+}^{S}$ or $\lambda_{-}^{S}$, respectively. Hence, for each $\lambda^{S}$ there is a system of coupled equations for the dark spinor components $\alpha$ and $\beta$ and also another coupled system for $\alpha^{*}$ and $\beta^{*}$, which are going to be solved separately. We denote now the first equation of each one of the systems below to be the equations related to $(\alpha, \beta)$, whereas the second ones regard $\left(\alpha^{*}, \beta^{*}\right)$. We can determine the above functions as

$$
\begin{aligned}
& \lambda_{+}^{S}: \\
& W_{1}(r)= \pm \int \sqrt{\frac{m^{2} F-\left(\omega-j \Omega_{H}+e A_{+}\right)^{2}}{F G}} d r \\
& W_{2}(r)= \pm \int \sqrt{\frac{\left(m \sqrt{F}+\omega-j \Omega_{H}+e A_{+}\right)^{2}}{F G}} d r
\end{aligned}
$$

$\lambda_{-}^{S}:$

$$
\left\{\begin{array}{l}
W_{3}(r)= \pm \int \sqrt{\frac{\left(m \sqrt{F}-\omega+j \Omega_{H}+e A_{+}\right)^{2}}{F G}} d r \\
W_{4}(r)=i W_{2}(r) .
\end{array}\right.
$$

For massless particles the solutions for $\lambda_{ \pm}^{S}$ are equivalent, being given by

$$
W_{2}(r)= \pm \int \sqrt{\frac{\left(\omega-j \Omega_{H}+e A_{+}\right)^{2}}{F G}} d r=-i W_{1}(r) .
$$

The above expression for $W_{2}(r)$ is similar to the results in [29] when $m=0$. The solution for $W_{2}(r)$ is provided by

$$
W_{2}(r)= \pm i \pi \frac{r_{+}^{2}-2 \beta r_{+}+a^{2}}{2\left(r_{+}+m\right)}\left(\omega-J \Omega_{H}+e A_{+}\right) .
$$

Since $F \rightarrow 0$ near the black hole horizon, the results hold both for massive and their massless limit particles as well. In fact, for such limit (28) and (29) are equivalent. It is worth emphasizing that (28) implies a real solution for $W_{1}(r)$ and thus a null contribution for the tunnelling effect.

In compliance with the WKB approximation, the tunnelling rate reads $\Gamma \propto \exp (-2 \operatorname{Im} I)$, where $I$ denotes the classical action for the path. Hence the imaginary part of the action becomes a prominent goal for the tunnelling process. The imaginary part of the action yields

$$
\operatorname{Im} I_{ \pm}= \pm i \pi \frac{r_{+}^{2}-2 \beta r_{+}+a^{2}}{2\left(r_{+}+m\right)}\left(\omega-J \Omega_{H}+e A_{+}\right) .
$$

Thus the resulting tunnelling probability is given by

$$
\begin{aligned}
\Gamma & =\frac{P_{(\text {emission })}}{P_{\text {(absorption) }}}=\frac{e^{-2 \operatorname{Im} I_{+}}}{e^{-2 \operatorname{Im} I_{-}}} \\
& =-2 \pi \frac{r_{+}^{2}-2 \beta r_{+}+a^{2}}{\left(r_{+}+m\right)}\left(\omega-J \Omega_{H}+e A_{+}\right) .
\end{aligned}
$$

Finally the Hawking temperature of the Kerr-Sen dilatonaxion black hole is acquired:

$$
T_{H}=\frac{1}{2 \pi} \frac{\left(r_{+}+m\right)}{r_{+}^{2}-2 \beta r_{+}+a^{2}}
$$


which is a universal formula also obtained by other methods for the Hawking temperature from fermions tunnelling [29]. Obviously when the parameter $a$ tends to zero, (33) provides the well-known Hawking radiation associated with the static black hole.

\section{Concluding Remarks}

We derived the Hawking radiation for spin- $1 / 2$ fermions of mass dimension one, represented by dark spinors tunnelling across a Kerr-Sen dilaton-axion black hole horizon. The temperature of these solutions was computed and demonstrated to confirm the universal character of the Hawking effect, even for mass dimension one fermions of spin- $1 / 2$ that are beyond the standard model. The mass dimension one feature of such spinor fields sharply suppresses the couplings to other fields of the standard model. Indeed, by power counting arguments, Elko spinor fields can self-interact and further interact with a scalar (Higgs) field, providing a renormalizable framework. This type of interaction means an unsuppressed quartic selfinteraction. The quartic self-interaction is essential to dark matter observations [30, 31]. Therefore, Elko spinor fields perform an adequate fermionic dark matter candidate. It can represent a real model for dark matter tunnelling across black holes. Corrections of higher order in $\hbar$ to the Hawking temperature (33) of type $I=I_{0}+\sum_{n \geq 1} \hbar^{n} I_{n}$ [11] can be still implemented in the context of mass dimension one spin$1 / 2$ fermions. Moreover, other mass dimension one fermions [20] and higher spin mass dimension one fermions can be studied in the context of black hole tunnelling methods; however these issues are beyond the scope of this paper, which comprised dark particles tunnelling across Kerr-Sen dilaton-axion black holes.

\section{Conflict of Interests}

The authors declare that there is no conflict of interests regarding the publication of this paper.

\section{Acknowledgments}

R. T. Cavalcanti thanks CAPES and UFABC. Roldão da Rocha is grateful to CNPq Grants no. 303027/2012-6, no. 451682/2015-7, and no. 473326/2013-2, for partial financial support, and to FAPESP Grant no. 2015/10270-0.

\section{References}

[1] P. Kraus and F. Wilczek, "Self-interaction correction to black hole radiance," Nuclear Physics B, vol. 433, no. 2, pp. 403-420, 1995.

[2] M. K. Parikh and F. Wilczek, "Hawking radiation as tunneling," Physical Review Letters, vol. 85, no. 24, pp. 5042-5045, 2000.

[3] K. Srinivasan and T. Padmanabhan, "Particle production and complex path analysis," Physical Review D, vol. 60, no. 2, Article ID 024007, 1999.

[4] M. Agheben, M. Nadalini, L. Vanzo, and S. Zerbini, "Hawking radiation as tunneling for extremal and rotating black holes," Journal of High Energy Physics, vol. 2005, article 014, 2005.
[5] M. Arzano, A. J. Medved, and E. C. Vagenas, "Hawking radiation as tunneling through the quantum horizon," Journal of High Energy Physics, vol. 2005, no. 9, p. 37, 2005.

[6] Q.-Q. Jiang, S.-Q. Wu, and X. Cai, "Hawking radiation as tunneling from the Kerr and Kerr-Newman black holes," Physical Review D, vol. 73, no. 6, Article ID 064003, 2006.

[7] J. Zhang and Z. Zhao, "Charged particles' tunnelling from the Kerr-Newman black hole," Physics Letters B, vol. 638, no. 2-3, pp. 110-113, 2006.

[8] R. Li, J.-R. Ren, and S.-W. Wei, "Hawking radiation of Dirac particles via tunneling from the Kerr black hole," Classical and Quantum Gravity, vol. 25, no. 12, Article ID 125016, 2008.

[9] L. Vanzo, G. Acquaviva, and R. Di Criscienzo, “Tunnelling methods and Hawking's radiation: achievements and prospects," Classical and Quantum Gravity, vol. 28, no. 18, Article ID 183001, 2011.

[10] R. Kerner and R. B. Mann, "Fermions tunnelling from black holes," Classical and Quantum Gravity, vol. 25, no. 9, Article ID 095014, 2008.

[11] R. Banerjee and B. R. Majhi, "Hawking black body spectrum from tunneling mechanism," Physics Letters B, vol. 675, no. 2, pp. 243-245, 2009.

[12] R. da Rocha and J. M. Hoff da Silva, "Hawking radiation from Elko particles tunnelling across black-strings horizon," Europhysics Letters, vol. 107, no. 5, Article ID 50001, 2014.

[13] D. Y. Chen, Q. Q. Jiang, and X. T. Zu, "Fermions tunnelling from the charged dilatonic black holes," Classical and Quantum Gravity, vol. 25, Article ID 205022, 2008.

[14] A. Sen, "Rotating charged black hole solution in heterotic string theory," Physical Review Letters, vol. 69, no. 7, pp. 1006-1009, 1992.

[15] A. Yale and R. B. Mann, "Gravitinos tunneling from black holes," Physics Letters B, vol. 673, no. 2, pp. 168-172, 2009.

[16] D. V. Ahluwalia-Khalilova and D. Grumiller, "Spin-half fermions with mass dimension one: theory, phenomenology, and dark matter," Journal of Cosmology and Astroparticle Physics, vol. 2005, no. 02, 12 pages, 2005.

[17] D. V. Ahluwalia, "On a local mass dimension one Fermi field of spin one-half and the theoretical crevice that allows it," http://arxiv.org/abs/1305.7509.

[18] D. V. Ahluwalia, C. Y. Lee, D. Schritt, and T. F. Watson, "Elko as self-interacting fermionic dark matter with axis of locality," Physics Letters B, vol. 687, no. 2-3, pp. 248-252, 2010.

[19] R. da Rocha, L. Fabbri, J. M. H. da Silva, R. T. Cavalcanti, and J. A. Silva-Neto, "Flag-dipole spinor fields in ESK gravities," Journal of Mathematical Physics, vol. 54, no. 10, Article ID 102505, 2013.

[20] R. T. Cavalcanti, "Classification of singular spinor fields and other mass dimension one fermions," International Journal of Modern Physics D, vol. 23, no. 14, Article ID 1444002, 2014.

[21] A. E. Bernardini and R. da Rocha, "Dynamical dispersion relation for ELKO dark spinor fields," Physics Letters B, vol. 717, no. 1-3, pp. 238-241, 2012.

[22] J. M. Hoff da Silva and R. da Rocha, "Unfolding physics from the algebraic classification of spinor fields," Physics Letters B, vol. 718, no. 4-5, pp. 1519-1523, 2013.

[23] A. G. Cohen and S. L. Glashow, "Very special relativity," Physical Review Letters, vol. 97, no. 2, Article ID 021601, 3 pages, 2006.

[24] C. G. Böhmer, J. Burnett, D. F. Mota, and D. J. Shaw, "Dark spinor models in gravitation and cosmology," Journal of High Energy Physics, vol. 2010, no. 7, article 053, 2010. 
[25] R. da Rocha, A. E. Bernardini, and J. M. da Silva, "Exotic dark spinor fields," Journal of High Energy Physics, vol. 2011, no. 4, article 110, 2011.

[26] A. P. dos Santos Souza, S. H. Pereira, and J. F. Jesus, "A new approach on the stability analysis in ELKO cosmology," The European Physical Journal C, vol. 75, no. 1, article 36, 2015.

[27] J. H. Horne and G. T. Horowitz, "Rotating dilaton black holes," Physical Review D, vol. 46, no. 4, pp. 1340-1346, 1992.

[28] G. W. Gibbons and K.-I. Maeda, "Black holes and membranes in higher-dimensional theories with dilaton fields," Nuclear Physics B, vol. 298, no. 4, pp. 741-775, 1988.

[29] D.-Y. Chen and X.-T. Zu, "Hawking radiation of fermions for the Kerr-Sen dilaton-axion black hole," Modern Physics Letters A, vol. 24, no. 14, pp. 1159-1165, 2009.

[30] P. K. Samal, R. Saha, P. Jain, and J. P. Ralston, "Signals of statistical anisotropy in WMAP foreground-cleaned maps," Monthly Notices of the Royal Astronomical Society, vol. 396, no. 1, pp. 511-522, 2009.

[31] M. Frommert and T. A. Ensslin, "The axis of evil-a polarization perspective," Monthly Notices of the Royal Astronomical Society, vol. 403, no. 4, pp. 1739-1748, 2010. 

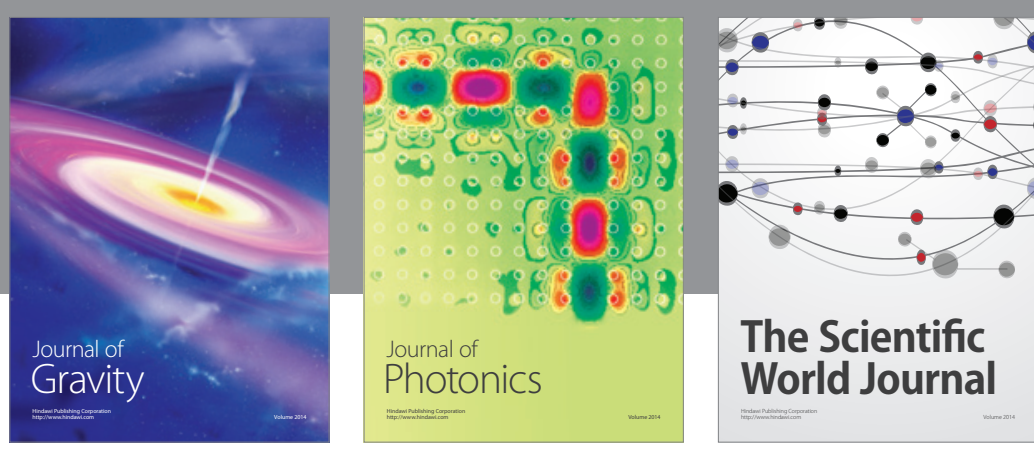

The Scientific World Journal
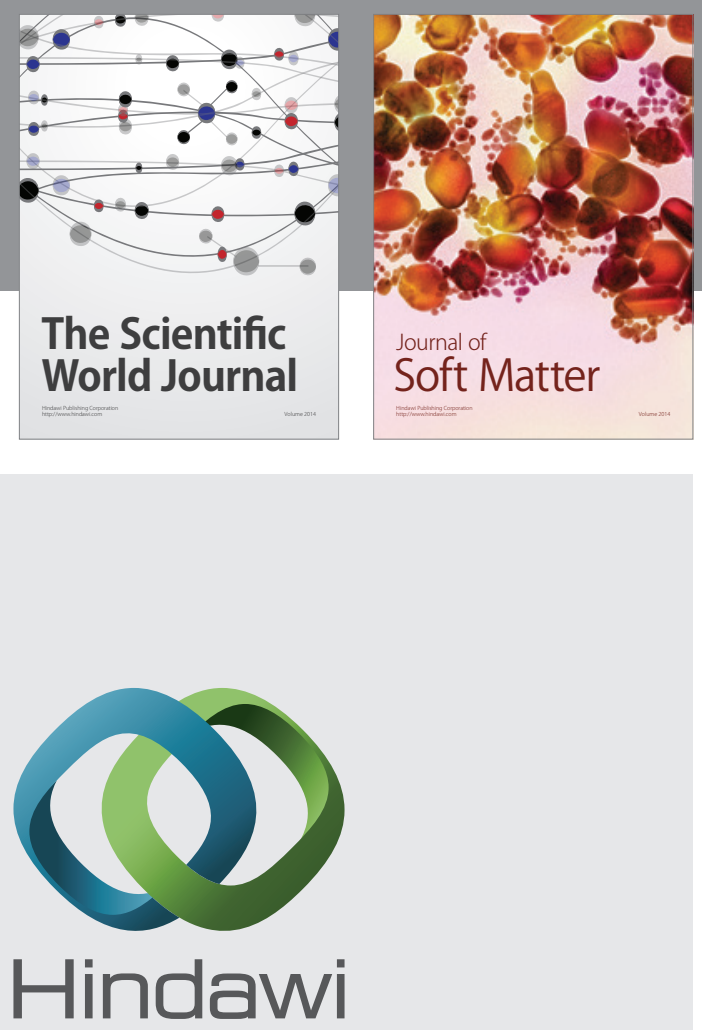

Submit your manuscripts at

http://www.hindawi.com

nternational Journal of

Statistical Mechanics
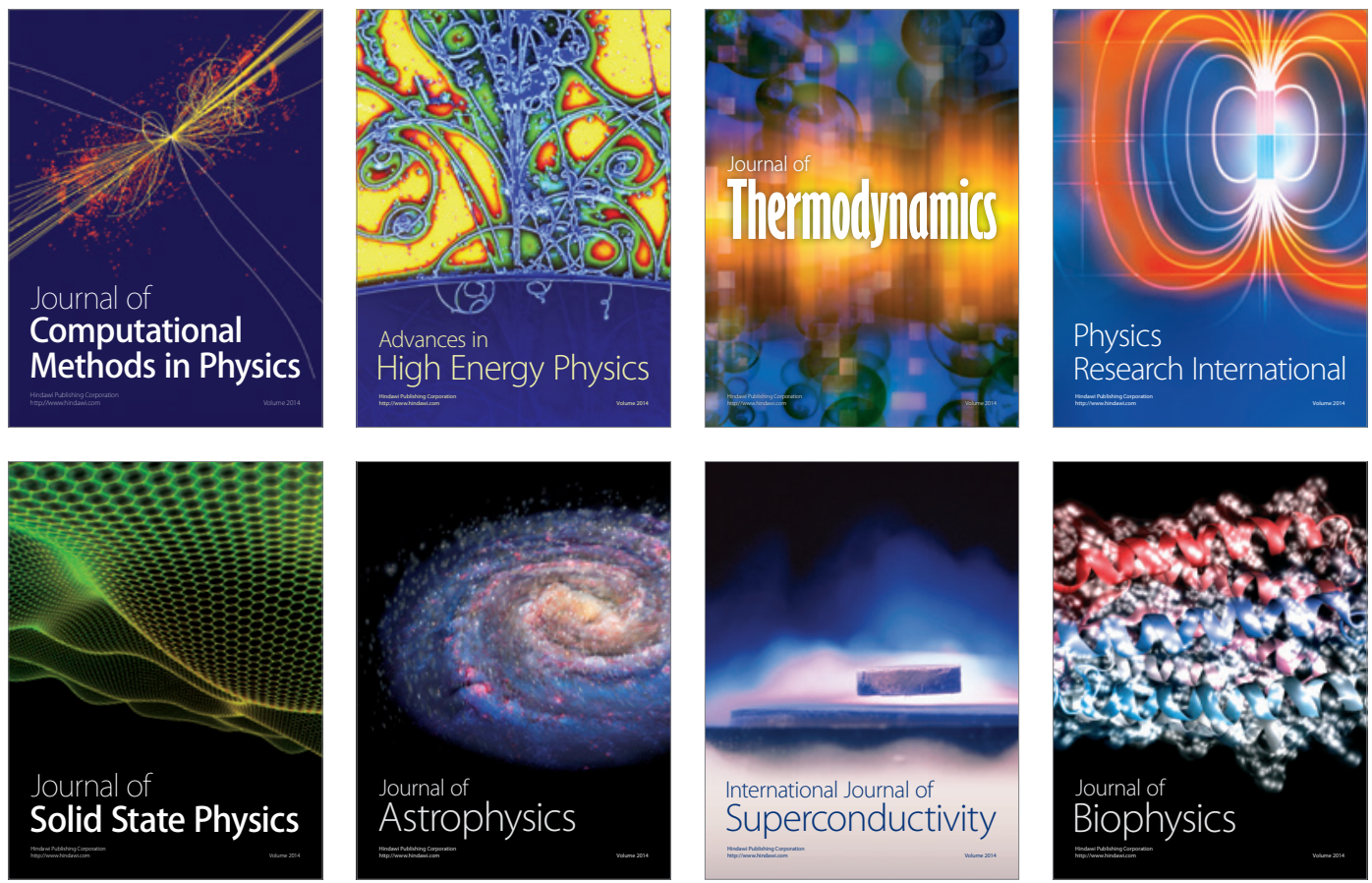
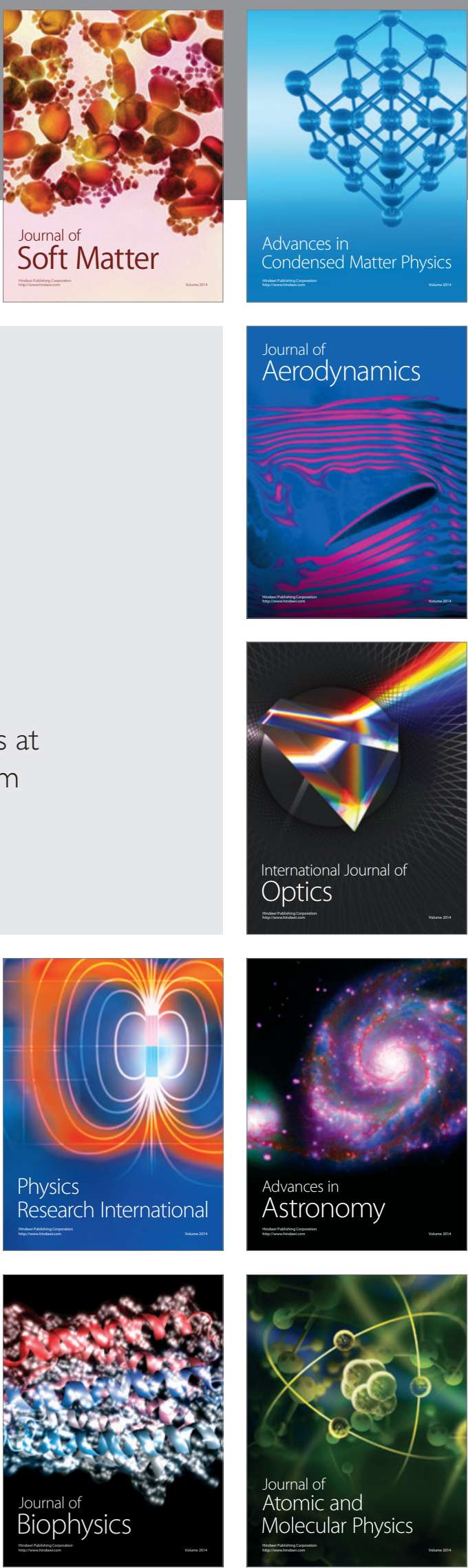